\title{
A History of Depression in Women is Associated with an Altered GABAergic Neuroactive Steroid Profile
}

\author{
Susan S. Girdler ${ }^{\star}$, Monica Lindgren, Patrizia Porcu, D.R. Rubinow, J. L. Johnson, and A. \\ Leslie Morrow \\ Department of Psychiatry, University of North Carolina at Chapel Hill
}

\begin{abstract}
The $3 \alpha, 5 \alpha-$ and $3 \alpha, 5 \beta$-reduced metabolites of progesterone, deoxycorticosterone, and dehydroepiandrosterone (DHEA) have potent effects on neurotransmission mediated by $\mathrm{GABA}_{\mathrm{A}}$ receptors, and dysregulation of these receptors has been implicated in depression. Using gas chromatography-mass spectrometry, we compared neuroactive steroid concentrations in women with a history of depressive disorders, but who were in full remission at the time of testing $(n=11)$ to never depressed women $(n=17)$ both before and after a challenge with oral micronized progesterone $(300 \mathrm{mg})$. Serum concentrations of the following were obtained: four progesteronederived GABAergic neuroactive steroids, the precursor pregnenolone, androstenedione-derived neuroactive steroids, and the precursor DHEA. As an index of conversion of progesterone to neuroactive steroids, we also examined ratios of neuroactive steroids to progesterone following the oral progesterone challenge. Results indicated that both before and after oral progesterone, women with histories of depression showed lower concentrations of all GABAergic neuroactive steroids than never depressed women. Those with a history of depression also tended to have lower cortisol concentrations. Because serum neuroactive steroids are mainly synthesized in the adrenals, we hypothesize that histories of depression may be associated with persistent adrenal suppression. Following the progesterone challenge, ratios of the progesterone-derived neuroactive steroids to plasma progesterone concentrations were elevated in women with depression histories, suggesting there may be an adaptive shift in the metabolism of progesterone that compensates for lower circulating neuroactive steroid concentrations.
\end{abstract}

\section{Keywords}

GABA; neurosteroids; depression; histories of depression

\section{Introduction}

Neuroactive steroids are endogenous neuromodulators, synthesized de novo in brain as well as in the adrenals and gonads. They have potent effects on neurotransmission mediated by $\mathrm{GABA}_{\mathrm{A}}$ receptors (Paul \& Purdy, 1992). The $3 \alpha, 5 \alpha$ - and $3 \alpha, 5 \beta$-reduced metabolites of progesterone, deoxycorticosterone, and dehydroepiandrosterone (DHEA) (Biggio \& Purdy, 2001; Frye, Duncan, Basham, \& Erskine, 1996; Majewska, Harrison, Schwartz, Barker, \&

\footnotetext{
(C) 2011 Elsevier Ltd. All rights reserved.

*Corresponding Author: Susan S. Girdler, Ph.D., University of North Carolina at Chapel Hill, CB\#7175, Medical School Wing D, Chapel Hill, NC 27599-7175. susan_girdler@med.unc.edu; Phone: 919-966-2179; FAX: 919-966-0708.

Publisher's Disclaimer: This is a PDF file of an unedited manuscript that has been accepted for publication. As a service to our customers we are providing this early version of the manuscript. The manuscript will undergo copyediting, typesetting, and review of the resulting proof before it is published in its final citable form. Please note that during the production process errors may be discovered which could affect the content, and all legal disclaimers that apply to the journal pertain.
} 
Paul, 1986) induce potent GABAergic actions, including anxiolytic, anticonvulsant, sedative/hypnotic and anti-nociceptive effects (Biggio \& Purdy, 2001; Frye, et al., 1996; Frye, Walf, Rhodes, \& Harney, 2004; Kavaliers \& Wiebe, 1987; Morrow, 2007) - actions, consistent with an integrated and adaptive response to stress (Purdy, Morrow, Moore, \& Paul, 1991). In addition to these adaptive behavioral effects of neuroactive steroids, animal models provide strong and consistent evidence that these steroid metabolites also act as endogenous suppressors of the hypothalamic-pituitary-adrenal (HPA)-axis following stress (Barbaccia et al., 1997; Purdy, et al., 1991; Strous, Maayan, \& Weizman, 2006). Although concentrations of neuroactive steroids in brain are somewhat higher than in plasma, plasma concentrations are likely to reflect brain concentrations because these neuroactive steroids can readily cross the blood brain barrier (Paul \& Purdy, 1992; Purdy, et al., 1991) and are likely to play important physiological roles.

For example, there is robust evidence from both animal and human studies that the $3 \alpha-$ reduced neuroactive steroid metabolites of progesterone, $(3 \alpha, 5 \alpha)$-3-hydroxypregnan-20-one ( $3 \alpha, 5 \alpha$-THP or allopregnanolone) and ( $3 \alpha, 5 \beta)$-3-hydroxypregnan-20-one ( $3 \alpha, 5 \beta$-THP) are decreased in depression (Eser et al., 2006; Nappi et al., 2001; Romeo et al., 1998; Strohle et al., 2000; Strohle et al., 1999; Uzunova, Sampson, \& Uzunov, 2006; Uzunova et al., 1998). When compared with non-depressed controls, depressed patients have lower plasma and CSF concentrations of 3 $\alpha, 5 \alpha$-THP (Eser, et al., 2006; Nappi, et al., 2001; Romeo, et al., 1998; Strohle, et al., 2000; Strohle, et al., 1999; Uzunova, et al., 2006; Uzunova, et al., 1998), but higher concentrations of the $3 \alpha, 5 \alpha$-metabolite of deoxycorticosterone ( $3 \alpha$, $5 \alpha$ )-3,21-dihydroxypregnan-20-one ( $3 \alpha, 5 \alpha$-THDOC) (Strohle, et al., 2000; Strohle, et al., 1999), which may reflect differential alteration in the biosynthesis of deoxycorticosterone or its metabolites in depression (Strohle, et al., 2000; Strohle, et al., 1999). Our prior research also suggested there may be a differential regulation of metabolic pathways involved in the conversion of progesterone to $3 \alpha, 5 \alpha$-THP in women with a menstrually related depressive disorder (premenstrual dysphoric disorder: PMDD), since those patients showed significantly elevated $3 \alpha, 5 \alpha-\mathrm{THP} /$ progesterone ratios relative to non-affected controls (Girdler, Straneva, Light, Pedersen, \& Morrow, 2001; Klatzkin, Morrow, Light, Pedersen, \& Girdler, 2006a). Our prior studies were limited, however, by the absence of measurement of

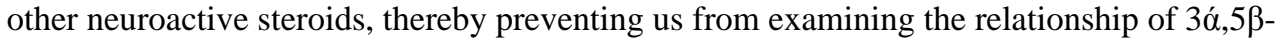
THP to other neurosteroids. Indeed, it is likely that the GABAergic metabolites of progesterone, deoxycorticosterone, and DHEA are both singularly and coordinately significant physiological regulators of central nervous system excitability (Morrow, 2007).

Regardless of mechanism, the pathophysiological relevance of altered neurosteroid concentrations in humans comes from studies showing inverse relationships between $3 \alpha, 5 \alpha-$ THP concentrations and severity of the depressive illness (Nappi, et al., 2001; Uzunova, et al., 1998) and from other studies showing that clinically efficacious treatment with

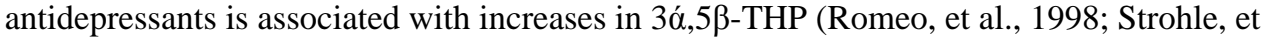
al., 2000; Strohle, et al., 1999; Uzunova, et al., 1998) and decreases in $3 \alpha, 5 \alpha$-THDOC) (Strohle, et al., 2000; Strohle, et al., 1999).

With our recent validation of a method using gas chromatography-mass spectrometry (GCMS) to simultaneously identify serum levels of $3 \alpha, 5 \alpha$ - and $3 \alpha, 5 \beta$-reduced derivatives of progesterone, deoxycorticosterone, DHEA and testosterone in both human and rat serum (Porcu et al., 2009), we report here a secondary analysis of the serum samples collected from women enrolled in a prior study that was designed to examine the metabolism of oral micronized progesterone to $3 \alpha, 5 \alpha-$ THP in women with PMDD and in non-PMDD controls (Klatzkin, et al., 2006a). That initial study assessed only $3 \alpha, 5 \alpha-$ THP, using radioimmunoassay techniques, but found that women with a history of depression (both 
PMDD and non-PMDD women) had lower $3 \alpha, 5 \alpha$-THP levels following oral micronized progesterone than never depressed women (Klatzkin, et al., 2006a).

In the present report, we sought to extend the previous literature on neuroactive steroid levels in depression, which has focused almost exclusively on the $3 \alpha, 5 \alpha$ - and $3 \alpha, 5 \beta$-reduced derivatives of progesterone (Uzunova, et al., 2006), by assessing a broader array of GABAergic neuroactive steroids in women with a history of depression compared with never depressed women (all non-PMDD women). Specifically, using GC-MS methods, we examined serum levels of four progesterone-derived GABAergic neuroactive steroids: $3 \alpha$, $5 \alpha$-THP, $3 \alpha, 5 \beta$-THP, $3 \alpha, 5 \alpha$-THDOC and ( $3 \alpha, 5 \beta)$-3,21-dihydroxypregnan-20-one ( $3 \alpha, 5 \beta$ THDOC), as well as the precursor pregnenolone. We also examined the androstenedione-

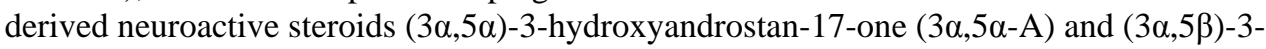
hydroxyandrostan-17-one ( $3 \alpha, 5 \beta-A)$, and the precursor DHEA (see Figure 1 for the biosynthetic pathway). Finally, we examined whether histories of depression would be associated with evidence for differential regulation of the metabolic pathways involved in the conversion of progesterone to its $3 \alpha, 5 \alpha$ - or $3 \alpha, 5 \beta$-neuroactive steroids following a controlled dose of oral micronized progesterone.

\section{Methods}

\section{Subjects}

Eleven women meeting DSM-IV criteria for prior depressive disorders and 17 controls with no lifetime history of depression comprised the subjects of this report. All subjects were medically healthy and free of any current psychiatric Axis I disorder. Normal ovulatory function was confirmed with the detection of the LH surge via home urine testing during the two preceding menstrual cycles, as well as with serum estradiol and progesterone values sampled during the luteal phase over three consecutive cycles in order to establish normal luteal phase concentrations. No participant had taken any prescription medication, including oral contraceptives, for at least three months prior to enrollment. The protocol was approved by the University of North Carolina, Committee on Protection for the Rights of Human Subjects. Subjects provided written informed consent before participation and each received $\$ 200$ compensation.

\section{Procedures}

Psychiatric histories: Structured clinical interviews (SCID) based on DSM-IV criteria for Axis I disorders were conducted during the follicular phase of the menstrual cycle by a trained interviewer. All diagnoses were based on a consensus diagnostic session with a psychiatrist. A history of psychotic disorders, bipolar disorder, drug or alcohol dependence, or PMDD was exclusionary for all subjects in this report. Eleven women were classified as having a history of depression, defined as prior major depressive disorder $(n=6)$, prior minor depressive disorder $(n=4)$ or prior adjustment disorder with depressed mood $(n=1)$. For past depressive disorders, seven months in full remission was required before testing. The never depressed control subjects were specifically recruited to have no lifetime history of any depressive disorder. For other Axis I disorders, three years in full remission was required for both groups.

Experimental Procedures: Each woman was tested during days 2-6 of the follicular phase on two consecutive menstrual cycles, a time associated with low and stable levels of endogenous progesterone and estradiol. Subjects completed the Beck Depression Inventory (Beck, Ward, Mendelson, Mock, \& Erbaugh, 1961) during the 24-hour period that preceded each laboratory test session in order to confirm the absence of clinically significant depressive symptoms at the time of testing. Using a double-blind, cross-over design, during 
one menstrual cycle subjects received $300 \mathrm{mg}$ of micronized progesterone while in the other they received a placebo in masked capsules, with order randomized. Since progesteronederived neuroactive steroid concentrations in the follicular phase are undetectable in a substantial portion of women (Girdler, et al., 2001), neurosteroid concentrations from the placebo session were not analyzed, though the placebo session served as a control condition for the non-specific effects of ingesting a pill or medication. Choice of dose was based both on our own pilot testing that revealed significant sedation with higher doses and our desire to induce plasma progesterone concentrations consistent with luteal phase concentrations (Freeman, Weinstock, Rickels, Sondheimer, \& Coutifaris, 1992).

The subjects arrived at the laboratory at 0700 after an overnight fast. Each consumed a standardized meal prepared by the institution's General Clinical Research Center containing 681 calories and composed of $18 \%$ protein, $22 \%$ fat and $60 \%$ carbohydrate. Subjects were required to consume the meal within $15 \mathrm{~min}$, immediately after which endogenous levels of serum progesterone and GABAergic neuroactive steroids were assessed. This sample constituted the pre-progesterone concentrations. At precisely $30 \mathrm{~min}$ following completion of the meal, subjects consumed three masked pills ( $300 \mathrm{mg}$ of micronized progesterone or placebo), followed by a 45-min rest period. An intravenous (i.v.) line was then established in an arm vein. Based on prior studies indicating that $300 \mathrm{mg}$ oral micronized progesterone results in peak plasma concentrations within 1-3 h following administration (McAuley, Kroboth, \& Kroboth, 1996), but remain relatively stable over 4 h (Freeman, et al., 1992), blood was sampled at $160 \mathrm{~min}$ following the administration of progesterone. This sample yielded the progesterone and neuroactive steroid concentrations reported here. This sample was also used to analyze resting cortisol concentrations as an additional measure of adrenal function. Moreover, because one aim of the parent study was to examine $3 \alpha, 5 \alpha$-THP responses to stress (Klatzkin, Morrow, Light, Pedersen, \& Girdler, 2006b), following the extended rest period that provided the 160 min samples, subjects were exposed to the Trier Social Stress Test (TSST) (Kirschbaum, Pirke, \& Hellhammer, 1993), involving the administration of a speech stressor and mental arithmetic stressor. After a 10-min recovery that followed the TSST, blood was again sampled and provides a measure of stress-induced cortisol concentrations.

Neuroactive Steroid Analysis-Neuroactive steroids were measured in serum by GCMS following purification by solid phase extraction, as previously described (Porcu, et al., 2009). Serum samples $(300 \mu \mathrm{l})$ were spiked with $400 \mathrm{pg} / \mathrm{ml}$ of each deuterated internal standard and applied to C18 solid phase extraction columns (RPN1910, $500 \mathrm{mg}$, GE Healthcare, UK) that had been preconditioned with $4 \mathrm{ml}$ methanol and $4 \mathrm{ml}$ distilled water. The column containing the sample was washed with $4 \mathrm{ml}$ distilled water in order to remove high polar impurities. Columns were dried under vacuum for 30 minutes and neuroactive steroids were then eluted with $2 \mathrm{ml}$ methanol. The extracts were evaporated in a speed vacuum concentrator (Thermo Fisher Scientific, Inc., Waltham, MA, USA). The dry residue was resuspended in $2 \mathrm{ml}$ of ethyl acetate/methanol $(80 / 20, \mathrm{v} / \mathrm{v})$ and the sample was filtered through a $\mathrm{NH}_{2}$ column (Supelclean $\mathrm{LC}-\mathrm{NH}_{2}, 500 \mathrm{mg}$, Supelco, Bellefonte, PA, USA) previously preconditioned with $4 \mathrm{ml}$ of ethyl acetate and $4 \mathrm{ml}$ of ethyl acetate/methanol $(80 / 20, v / v)$. The neuroactive steroids passed unretained through the sorbent, and the eluate was collected. The $\mathrm{NH}_{2}$ column was further rinsed with $2 \mathrm{ml}$ of the solvent mixture and the combined eluates were evaporated in the speed vacuum concentrator. Dried samples after purification were resuspended in $800 \mu \mathrm{l}$ methanol and transferred to derivatization vials (Wheaton, Millville, NJ, USA). Methanol was evaporated and samples were derivatized in $450 \mu 1$ of ethyl acetate and $50 \mu 1$ of heptafluorobutyric acid anhydride, followed by vortex mixing. Samples were allowed to react for two hours at room temperature and were dried under a gentle stream of nitrogen. Derivatized samples were resuspended in $10 \mu 1$ of heptane and $2 \mu 1$ of each sample was injected in duplicate into the GC-MS. Analysis was carried out 
on an Agilent 6890 gas chromatograph coupled to a 5973 mass selective detector (Agilent Technologies, Inc., Santa Clara, CA, USA) operated in negative chemical ionization mode. A capillary column $(30 \mathrm{~m} \times 0.25 \mathrm{~mm}, 0.25 \mu \mathrm{m}$ film thickness, $5 \%$-phenylmethylpolysiloxane, J\&W Scientific, Agilent Technologies, Inc., Santa Clara, CA, USA) was used to separate the derivatives of each neuroactive steroid. Samples were injected into the GC in splitless mode at $12 \mathrm{psi}$ and at $250^{\circ} \mathrm{C}$ using a 7683 series injector (Agilent Technologies, Inc., Santa Clara, CA, USA). The carrier gas was ultrapure helium (99.9995\%, Airgas National Welders, Durham, NC, USA) set at constant flow of $1.0 \mathrm{ml} /$ min. Methane (99.999\% Airgas National Welders, Durham, NC, USA) was the reagent gas. The initial GC oven temperature was $75^{\circ} \mathrm{C}(0.86 \mathrm{~min}$ hold $)$, followed by an increase to $210^{\circ} \mathrm{C}$ at $35^{\circ}$ increments ( $3 \mathrm{~min}$ hold), an increase to $235^{\circ} \mathrm{C}$ at $2.5^{\circ}$ increments ( 9 min hold) and finally to $310^{\circ} \mathrm{C}$ at $25^{\circ}$ increments ( 2 min hold). The transfer line temperature was maintained at $280^{\circ} \mathrm{C}$. Neuroactive steroids were analyzed by single ion monitoring. The data acquisition was broken into retention windows corresponding to the elution of the different neuroactive steroid groups. The temperatures of mass spectrometer source and quadrupole were $150^{\circ} \mathrm{C}$. Neuroactive steroids were quantified by interpolation of linear regression standard curves. Calibration curves were made in $300 \mu \mathrm{l}$ distilled water spiked with $5 \mu \mathrm{l}$ human charcoal-stripped serum (Gemini Bio-Products, Woodland, CA, USA), with 400 pg/ $\mathrm{ml}$ of each deuterated internal standard and with the appropriate known concentration of neuroactive steroids $(2,10,20,50,100,200,500,1000,2000$ and $3000 \mathrm{pg} / \mathrm{ml})$. A blank standard ( $5 \mu 1$ human charcoal-stripped serum/300 $\mu$ l distilled water) was also included. Calibration curves underwent the same extraction procedure as the samples.

Steroid standards (>99\% purity) for 3 $\alpha, 5 \alpha-\mathrm{A}, 3 \alpha, 5 \beta-\mathrm{A}, 3 \alpha, 5 \beta-\mathrm{THP}, 3 \alpha, 5 \beta$-THDOC, DHEA and pregnenolone were purchased from Steraloids, Inc. (Newport, RI, USA). Deuteriumlabeled standards (>95\% purity) (d4-17,21,21,21)-3 $\alpha, 5 \alpha$-THP and (d3-17,21,21)-3 $\alpha, 5 \alpha-$ THDOC were purchased from Cambridge Isotope Laboratories, Inc. (Andover, MA, USA). (d4-2,2,4,4)-3 $\alpha, 5 \alpha-A$ was purchased from Cerilliant (Round Rock, TX, USA). 3 $\alpha, 5 \alpha-$ THP, $3 \alpha, 5 \alpha$-THDOC and (d4-17,21,21,21)-pregnenolone (98\% purity) were synthesized by Dr. R.H. Purdy (Veterans Medical Research Foundation, San Diego, CA, USA). Derivatization reagent heptafluorobutyric acid anhydride was purchased from Pierce (Rockford, IL, USA). Organic solvents were pesticide grade from Thermo Fisher Scientific, Inc. (Waltham, MA, USA).

Data Analysis-Group differences in demographic factors and psychiatric histories were examined using a one-way (history of depression: yes or no) analysis of variance (ANOVA) or chi-square analysis.

Descriptive statistics and plots (not shown) were produced for the concentration of each GABAergic neuroactive steroid before and after oral progesterone. As expected, based in part on the considerable intersubject variability in the extent of progesterone absorbed after administration of oral micronized progesterone (McAuley, et al., 1996), there was extreme positive skewness in the distributions of the neuroactive steroids. Consequently, standard normal theory ANOVA methods could not be used for inference. In order to examine diagnosis-related differences in GABAergic neuroactive steroid concentrations before and after oral progesterone, a generalized estimating equations (GEE) model with a gamma distribution and log link function was fit. Depression group, neuroactive steroid $(3 \alpha, 5 \alpha-$ THP, $3 \alpha, 5 \beta$-THP, pregnenolone, $3 \alpha, 5 \alpha-\mathrm{A}, 3 \alpha, 5 \beta-\mathrm{A}$ and DHEA), time point (pre- versus post-progesterone administration), and all two and three way interactions were included in the model as fixed effects. The within subject correlation due to multiple neuroactive steroids and time points was estimated using empirical standard errors (Zeger, Liang, \& Albert, 1988). Goodness of fit statistics were examined to indicate adequate fit of the model. Any non-significant interaction terms were deleted before performing hypothesis tests in the 
final model. To determine the source of any significant depression or time effect, a separate GEE model was fit to each neurosteroid. Such models were identical to the model described above, but excluded fixed effects terms and interactions for neurosteroid. Progesterone and cortisol were also examined at this stage using a GEE model with a gamma distribution. Due to the non-detection of pre-progesterone concentrations of $3 \alpha, 5 \alpha$-THDOC and $3 \alpha, 5 \beta-$

THDOC in the majority of subjects, these neuroactive steroids were not included in the GEE analyses. Thus, $3 \alpha, 5 \alpha$-THDOC and $3 \alpha, 5 \beta$-THDOC were considered only for analyses involving ratios of neuroactive steroids to progesterone at the post-progesterone time point (see below).

Next, correlational analyses were performed in the full sample of women $(n=28)$ in order to examine the relationship of endogenous progesterone concentrations to neuroactive steroid concentrations as well as inter-correlations among the various neurosteroids both before and after oral micronized progesterone.

Finally, as an index of the metabolism of oral micronized progesterone to neuroactive steroids (Girdler, et al., 2001), for the neuroactive steroids most directly derived from progesterone ( $3 \alpha, 5 \alpha$-THP, $3 \alpha, 5 \beta$-THP, $3 \alpha, 5 \alpha$-THDOC and $3 \alpha, 5 \beta$-THDOC), we examined diagnosis-related differences in the ratios of the GABAergic neuroactive steroids to progesterone using one-way ANOVAs (history of depression: yes or no). All ratios were $\log$-transformed to aid in the interpretability of the graphical representation.

For certain neurosteroids, there were individual sample values that exceeded three standard deviations above the mean (11 samples total), most likely reflecting sample contamination. These values were not included in the analyses. Consequently, the sample size varies slightly across the different neurosteroids. See Table 2 for the sample size that contributed to each neurosteroid concentration value.

All analyses were conducted using SAS software, version 9.2

\section{Results \\ Demographic factors}

As summarized in Table 1, there were no differences between never depressed women and those with histories of depression in age, body mass index (BMI), or proportion of minorities. There were no differences between groups in the proportion of subjects with prior posttraumatic stress disorder (PTSD) or eating disorders, though more women with prior depression had a history of anxiety $\left(\mathrm{X}^{2}(1)=4.9, \mathrm{p}<.05\right)$ and substance abuse $\left(\mathrm{X}^{2}(1)=\right.$ $3.9, \mathrm{p}<.05)$ than never depressed women. Women with a history of depression also had elevated Beck Depression Inventory scores at the time of testing than never depressed women $(\mathrm{F}(1,26)=5.47, \mathrm{p}<.05)$. For those with a history of depression, Table 1 also summarizes the mean number of months since the last depressive episode and the proportion of women with single versus multiple episodes.

\section{Progesterone concentrations at baseline and following oral progesterone administration}

Table 2 summarizes progesterone and neuroactive steroid concentrations before and after oral micronized progesterone administration. For all women, progesterone serum levels were elevated following oral progesterone $(\mathrm{F}(1,27)=133.6, \mathrm{p}<0.0001)$. However, as previously reported in the prior study from which we conducted these secondary analyses (Klatzkin, et al., 2006a), women with prior depression had lower serum progesterone concentrations both before and after oral micronized progesterone compared to women with no prior depression $(\mathrm{F}(1,26)=10.47, \mathrm{p}<0.01)$. 


\section{Neuroactive steroid concentrations at baseline and following oral progesterone administration}

As summarized in Table 2, at both pre- and post-oral progesterone time points, women with histories of depression exhibited lower levels of all neuroactive steroids than did never depressed women $(\mathrm{F}(1,26)=12.2, \mathrm{p}<0.01)$. A significant time point $\times$ neuroactive steroid interaction was also obtained $(\mathrm{F}(5,127)=37.96, \mathrm{p}<0.0001)$. In order to determine the source of the interaction a separate GEE model was fit to each neurosteroid. Progesterone induced a significant increase in $3 \alpha, 5 \alpha-\mathrm{THP}(\mathrm{F}(1,26)=125.5, \mathrm{p}<0.0001)$ and $3 \alpha, 5 \beta-\mathrm{THP}$ $(\mathrm{F}(1,27)=8.66, \mathrm{p}<0.01)$, but did not increase pregnenolone, DHEA, $3 \alpha, 5 \alpha-\mathrm{A}$ or $3 \alpha, 5 \beta-\mathrm{A}$ (Table 2). Although $3 \alpha, 5 \alpha-$ THDOC and $3 \alpha, 5 \beta$-THDOC were not included in the formal analyses due to so few subjects with detectable concentrations at the pre-progesterone time point (13 and 7 subjects, respectively), both $3 \alpha, 5 \alpha$-THDOC and $3 \alpha, 5 \beta$-THDOC were detectable in the majority of subjects at the post-progesterone time point (22 and 21 subjects, respectively), suggesting that oral progesterone did induce a significant increase in these neuroactive steroids as well.

\section{Cortisol concentrations at baseline rest and following mental stress}

While both groups showed a significant increase in cortisol following mental stress relative to their own baseline rest concentrations $(F(1,26)=6.44, p<0.02)$, women with histories of depression had reduced cortisol concentrations relative to never depressed women at both time points $(\mathrm{F}(1,26)=5.64, \mathrm{p}<0.03)$.

\section{Relationship between serum progesterone concentrations and neuroactive steroid concentrations before and after oral progesterone administration}

Serum progesterone concentrations before the oral administration of micronized were not significantly correlated with any of the neurosteroids $(\mathrm{rs}=-0.24-+0.29)$. In contrast, serum progesterone concentrations following oral progesterone administration were highly correlated with each of the progesterone-derived neuroactive steroids: $3 \alpha, 5 \alpha-\mathrm{THP}(\mathrm{r}=$ $+0.91, \mathrm{p}<.0001), 3 \alpha, 5 \beta-$ THP $(\mathrm{r}=+0.75, \mathrm{p}<.0001), 3 \alpha, 5 \alpha-$ THDOC $(\mathrm{r}=+0.766 \mathrm{p}<.001)$, and $3 \alpha, 5 \beta$-THDOC $(\mathrm{r}=+0.96, \mathrm{p}<.0001)$, as well as with the precursor pregnenolone $(\mathrm{r}=+0.54$, $\mathrm{p}<.01)$. Serum progesterone concentrations following oral progesterone were not correlated with DHEA $(\mathrm{r}=-0.20), 3 \alpha, 5 \alpha-\mathrm{A}(\mathrm{r}=+0.07)$ and $3 \alpha, 5 \beta-\mathrm{A}(\mathrm{r}=+0.07)$. These results strongly indicate that the significant increase in the progesterone-derived neuroactive steroids following oral progesterone administration resulted directly from the metabolism of progesterone to its derivative neuroactive steroids.

\section{Relationship among the neuroactive steroid concentrations before and after oral progesterone administration}

Both prior to and after progesterone administration, $3 \alpha, 5 \alpha$-THP, $3 \alpha, 5 \beta$-THP, pregnenolone, DHEA, $3 \alpha, 5 \alpha$-A and $3 \alpha, 5 \beta$-A were highly inter-correlated with one another ( $\mathrm{rs}=+0.41-$ +0.98 , ps $<0.05$ ), with the exception of pre-progesterone $3 \alpha, 5 \beta$-A that was not significantly correlated with pre-progesterone pregnenolone $(r=+0.31)$. For $3 \alpha, 5 \alpha-$ THDOC and $3 \alpha, 5 \beta$ THDOC, since pre-progesterone concentrations were undetectable in a substantial number of subjects, only post-progesterone correlations were examined, yielding high a intercorrelation with each other $(\mathrm{r}=+0.60, \mathrm{p}<.01)$, and with most other neuroactive steroids ( $\mathrm{rs}=$ $+0.47-+0.96$, ps <.05). The exceptions were $3 \alpha, 5 \beta$-THDOC with $3 \alpha, 5 \beta-\mathrm{A}(\mathrm{r}=+0.28 \mathrm{p}=\mathrm{ns})$ and with $3 \alpha, 5 \alpha-\mathrm{A}(\mathrm{r}=+0.35, \mathrm{p}=\mathrm{ns})$; and $3 \alpha, 5 \alpha-$ THDOC with $3 \alpha, 5 \beta-\mathrm{A}(\mathrm{r}=+0.17, \mathrm{p}=\mathrm{ns})$.

\section{Neuroactive steroid/Progesterone ratios}

$3 \alpha, 5 \alpha-T H P, 3 \alpha, 5 \beta-T H P, 3 \alpha, 5 \alpha-T H D O C$ and $3 \alpha, 5 \beta-T H D O C$-Prior to oral progesterone administration, women with prior depression did not differ from never 
depressed women in neuroactive steroid/progesterone ratios for $3 \alpha, 5 \alpha$-THP (6.1 vs. 5.7, respectively), $3 \alpha, 5 \beta$-THP (6.6 vs. 5.2), $3 \alpha, 5 \alpha$-THDOC (4.7 vs. 4.3 ) or $3 \alpha, 5 \beta$-THDOC (4.3 vs. 4.9) (all ps > .15). However, as illustrated in Figure 2, following progesterone administration, women with a history of depression had significantly higher ratios of $3 \alpha, 5 \alpha-$ THP $(\mathrm{F}(1,26)=4.84, \mathrm{p}<0.05), 3 \alpha, 5 \beta-$ THP $(\mathrm{F}(1,27)=6.98, \mathrm{p}<0.05)$ and $3 \alpha, 5 \alpha-$ THDOC $(\mathrm{F}(1,21)=8.58, \mathrm{p}<0.01)$ to progesterone than never depressed women. Women with prior depression also tended to have a higher ratio of $3 \alpha, 5 \beta$-THDOC/progesterone $(F(1,20)=4.1$, $\mathrm{p}<0.06)$ than the never depressed women at the post-progesterone time point.

\section{Discussion}

The primary finding of this study was a generalized reduction in GABAergic neuroactive steroid concentrations in women with histories of depression relative to never depressed women. This was true for the $3 \alpha, 5 \alpha$ - and $3 \alpha, 5 \beta$-derived neuroactive steroids of progesterone as well as its precursor pregnenolone, and for the $3 \alpha, 5 \alpha-$ and $3 \alpha, 5 \beta$-derived neuroactive steroids of androstenedione and their precursor DHEA. Moreover, this pattern of reduced neuroactive steroid concentrations was evident both before and after the administration of oral micronized progesterone. It should be noted that the $3 \alpha, 5 \alpha-$ and $3 \alpha, 5 \beta$-derived neuroactive steroids of deoxycorticosterone were also lower following oral micronized progesterone in women with depression histories, though the number of samples with nondetectable levels prior to the progesterone administration prevented formal statistical analyses of this effect.

The principal finding of reduced GABAergic neuroactive steroids in women with histories of depression is partially inconsistent with two prior reports in patients with current major depression that found lower $3 \alpha, 5 \alpha$-THP but elevated concentrations of $3 \alpha, 5 \alpha$-THDOC, suggesting a differentially greater conversion of progesterone to deoxycorticosterone in depressed patients, thereby reducing the substrate for $3 \alpha, 5 \beta$-THP and increasing it for $3 \alpha, 5 \beta$ THDOC (Strohle, et al., 2000; Strohle, et al., 1999). A number of methodological differences between our study and those of Strohle et al., (Strohle, et al., 2000; Strohle, et al., 1999) may account for the discrepant findings, not the least of which is that our study included remitted women with histories of depression, while the studies by Strohle and colleagues included patients with current depression. One theoretical possibility is that different phases of the depressive illness are associated with differences in enzymatic or other processes underlying the synthesis and metabolism of neuroactive steroids. Thus, current depressive illness may provide a physiologic state in which neurosteroid metabolism differs, similar to what has been observed in pregnancy (Gilbert Evans, Ross, Sellers, Purdy, $\&$ Romach, 2005). Additionally, the studies by Strohle and colleagues included either exclusively male (Strohle, et al., 2000) or mixed gender samples (Strohle, et al., 1999).

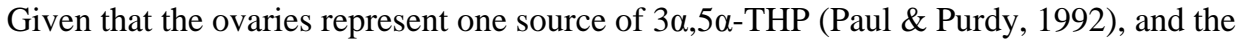
lack of control for menstrual cycle phase in their study that included women (Strohle, et al., 1999), gender or cycle phase could well play a role in depression-related differences in neuroactive steroid concentrations. Regardless of the reason for the discrepant findings, a remarkable feature of our results is the evidence for persistent disturbance in a variety of GABAergic neuroactive steroids concentrations in women who had been in remission from their depression for an average of five years.

While the reason for this global reduction in GABAergic neuroactive steroids in women with prior depression is unknown, the most parsimonious explanation may be one of adrenal suppression. The adrenals are the main secretory source of circulating progesterone during the early follicular phase, the time frame for testing in the present report, with the main source of progesterone not shifting to the ovaries until the late, pre-ovulatory follicular phase (De Geyter, De Geyter, Huber, Nieschlag, \& Holzgreve, 2002). Adrenocorticotropic 
hormone (ACTH) stimulates the conversion of cholesterol to pregnenolone in the adrenal cortex (Simpson \& Waterman, 1988), which is rapidly converted to progesterone, 17 $\alpha$ hydroxyprogesterone and androstenedione by the enzymes $3 \beta$-hydroxysteroid dehydrogenase and 17 $\alpha$-hydroxylase/17,20-lyase (cytochrome P450c17) (see Figure 1). Consequently, adrenal suppression could account for the pattern of our results since pregnenolone, the precursor for all of the GABAergic neuroactive steroids, was significantly lower in women with depression histories. Adrenal suppression is also supported, in part, by our observation that the women with histories of depression exhibited lower plasma cortisol concentrations at rest and following stress than the never depressed women.

While HPA-axis hyperactivity is considered a hallmark of melancholic depression (Gillespie \& Nemeroff, 2005), studies on HPA-axis activity associated with remission of depression are scarce and conflicting (Deuschle et al., 2003; Sachar, Hellman, Fukushima, \& Gallagher, 1970; Steiger, von Bardeleben, Herth, \& Holsboer, 1989). However, results of a recent study in women in full remission from major depression for an average of 61 months are also consistent with adrenal suppression since they found lower morning salivary cortisol concentrations, lower resting serum cortisol concentrations, and a blunted cortisol and ACTH response to mental stress in the patients with remitted depression compared with agematched never depressed women (Ahrens et al., 2008).

What might account for a decreased pituitary drive to the adrenals and suppression of steroidogenesis in women with depression histories? One possibility relates to the stressresponsive nature of the GABAergic neuroactive steroids. Under the control of ACTH (Reddy, 2006), the precursors DHEA (Maninger, Capitanio, Mason, Ruys, \& Mendoza) and prenenolone (Vallee, Rivera, Koob, Purdy, \& Fitzgerald, 2000) as well as their $3 \alpha, 5 \alpha$ - and $3 \alpha, 5 \beta$-metabolites of progesterone, deoxycorticosterone and androstenedione, have been shown to increase in response to acute stress (Barbaccia, et al., 1997; Paul \& Purdy, 1992). The most consistently studied of these neuroactive steroids within the context of stress are $3 \alpha, 5 \alpha$-THP and $3 \alpha, 5 \alpha$-THDOC. Animal models provide strong and consistent evidence that these steroid metabolites act as endogenous suppressors of the endocrine response to stress (Morrow, Devaud, Purdy, \& Paul, 1995; Purdy, et al., 1991), restoring both normal GABAergic and HPA-axis function following acute stress (Barbaccia, et al., 1997; Guo et al., 1995; Patchev, Hassan, Holsboer, \& Almeida, 1996; Strous, et al., 2006). However, a more recent animal study showed that $3 \alpha, 5 \alpha$-THP injection in the absence of stress produced transient increases in basal corticosterone, ACTH and corticotrophin releasing factor (CRF) levels (Naert, Maurice, Tapia-Arancibia, \& Givalois, 2007). Thus, $3 \alpha, 5 \alpha-$ THP may have a regulatory function that depends on the basal state of the animal (Naert, et al., 2007).

While neurohormonal activation in response to stress is adaptive in the short-term, long-term activation of such responses due to repeated or chronic stress may lead to persistent dysregulation in stress responsive systems. In humans, the price of repeated biological adaptations to stress has been termed allostatic load, referring to the long-term effect of physiologic responses to stress (McEwen, 1998). Allostatic load, or dysregulation in stress responsive systems, may manifest in a variety of ways, including repeated elevations of neurohormonal stress mediators (e.g., cortisol, norepinephrine) over long periods, as a failure to adapt to a stressor, as a failure to shut off the normal stress response, as alterations in basal neurohormonal concentrations, or as an inadequate hormonal response to stress (McEwen, 1998; Siever \& Davis, 1985). It has been suggested that such hypoactivation of stress responsive systems may result from a wearing out or exhaustion of the system due to long-term stress exposure and allostatic load (McEwen, 1998).

Although no studies to date have specifically examined the association of chronic stress with neuroactive steroid function in depressed patients, it could be argued that depression is itself 
a chronic stressor, and increased psychosocial stress is frequently a trigger for the onset of depressive illness (Monroe, 2005). The link between chronic stress and neuroactive steroid "depletion" is supported in part by animal models showing that protracted long-term adaptation to the stress of social isolation, a characteristic feature of human depression (Prince, Harwood, Blizard, Thomas, \& Mann, 1997; Roberts, Kaplan, Shema, \& Strawbridge, 1997), is associated with anxiety, aggression, and decreased response to GABAmimetic drugs and with significantly lower brain and plasma neuroactive steroid concentrations, including $3 \alpha, 5 \alpha$-THP and $3 \alpha, 5 \alpha$-THDOC (Dong et al., 2001; Serra et al., 2000). The finding that the expression in mouse brain of 5 $\alpha$-reductase (mRNA and protein), the rate limiting enzyme in the conversion of progesterone to $3 \alpha, 5 \alpha-\mathrm{THP}$, is down-regulated during protracted social isolation supports the view that chronic stress could alter neuroactive steroid synthesis (Reddy, 2006).

During the course of long-term or repeated depressions (58\% of our sample had recurrent depressions) the possibility exists that an initial HPA-axis overdrive would, over time, result in allostatic load as reflected in 'exhaustion' of the adrenal-axis system. A meta-analysis on HPA-axis activation associated with chronic stress in humans supports this supposition, since it found that while chronic stress is initially associated with HPA-axis hyperactivity, over time cortisol secretion is reduced by chronic stress exposure (Miller, Chen, \& Zhou, 2007). Animal studies also indicate that chronic stress exposure is associated with HPA-axis hyporesponsiveness to acute stressors (Blanchard, Sakai, McEwen, Weiss, \& Blanchard, 1993; McKittrick, Blanchard, Blanchard, McEwen, \& Sakai, 1995).

Thus, while our novel preliminary data suggest that there is a persistent generalized reduction in GABAergic neuroactive steroids in women with histories of depression, consistent with adrenal suppression, the argument for adrenal suppression would be strengthened by the inclusion of other measures of HPA-axis regulation. We hypothesize that the pattern of diminished GABAergic neuroactive steroid and cortisol concentrations in those with a history of depression may reflect a physiological adaptation resulting from depression and/or chronic stress. The absence of measures of psychosocial stress is a limitation of the present study and clearly precludes any definitive conclusions about psychosocial factors that may have contributed to the diminished neuroactive steroid profile in women with prior depression.

Residual depressive symptoms, even in the absence of current depressive illness could represent an additional mechanism contributing to the blunted GABAergic neuroactive steroid profile in the present cohort of women with depression histories since they had higher scores on the Beck Depression Inventory than the never depressed women at the time of testing. While their depressive symptom scores did not achieve levels typically considered to be of clinical relevance, nonetheless, the possibility remains that chronic, residual low levels of depressive symptoms following a depressive illness may contribute to sustained suppression of neuroactive steroid concentrations.

Alternatively, sustained and elevated depressive symptoms in those with a history of depression may result from their lower GABAergic neurosteroid concentrations, consistent with the paradoxical behavioral effect that has been observed for $\mathrm{GABA}_{\mathrm{A}}$ receptor modulators. For example, both animal and human studies have shown that GABA steroids (via the $\mathrm{GABA}_{\mathrm{A}}$ receptor complex in brain) exert both positive (e.g. anxiolytic, sedative, and anaesthetic properties) and negative behavioral effects (e.g. irritability, aggression, and depressive mood) (Andreen et al., 2009). A possible mechanism for this paradoxical effect is suggested by animal studies indicating that certain $\mathrm{GABA}_{\mathrm{A}}$ receptor modulators, including allopregnanolone, have biphasic effects, with low doses or concentrations increasing negative behavioral effects, and high doses or concentrations decreasing negative behavioral 
effects and having more positive behavioral effects (e.g. (Beauchamp, Ormerod, Jhamandas, Boegman, \& Beninger, 2000; Miczek, DeBold, van Erp, \& Tornatzky, 1997). Recent reports from human studies also indicate a bimodal effect of $\mathrm{GABA}_{\mathrm{A}}$ modulators since postmenopausal women receiving combined hormone therapy (estrogen plus a progestin) exhibit more intense adverse mood effects during treatment with lower doses of the progestin compared with higher doses (Andreen, Bixo, Nyberg, Sundstrom-Poromaa, \& Backstrom, 2003; Bjorn et al., 2002). Thus, in contrast to depression and chronic-stress associated depletion of GABAergic neurosteroid concentrations (via adrenal suppression) in women with histories of depression, the possibility exists that the lower neurosteroid concentrations serve as pathogenic triggers for the onset of depressive illness in vulnerable individuals and for the maintenance of depressive symptoms even after remission of the depressive episode.

Regardless of the mechanism underlying the reduced GABAergic neuroactive steroid concentrations, there appears to be a compensatory attempt of the system to adjust for lower circulating levels. This is suggested by our findings of elevated neuroactive steroid to progesterone ratios for $3 \alpha, 5 \alpha$-THP, $3 \alpha, 5 \beta-$ THP, $3 \alpha, 5 \alpha$-THDOC, and $3 \alpha, 5 \beta$-THDOC following the oral administration of progesterone in women with histories of depression relative to never depressed women. Specifically, in the women with prior depressions, for a given concentration of progesterone, there is evidence for a differentially greater increase in its conversion to neuroactive steroids. In two separate cohorts of women with PMDD, we have previously reported elevated $3 \alpha, 5 \alpha$-THP to progesterone ratios relative to non-PMDD controls (Girdler, et al., 2001; Klatzkin, et al., 2006a). These results, taken together, suggest that recurrent affective syndromes may be associated with residual alterations in the enzymatic processing of progesterone to its neuroactive steroid metabolites. However, it must also be acknowledged that one limitation to our study was that we assessed progesterone and neuroactive steroid concentrations at only one time point following oral micronized progesterone. The possibility exists that we may have seen a different pattern of results regarding diagnosis-related differences in GABAergic neuroactive steroid concentrations or in ratios of neuroactive steroids to progesterone at different time points in the metabolic process.

Given the stress-responsiveness of neuroactive steroids (Purdy, et al., 1991), their role in HPA-axis regulation (Morrow, et al., 1995) and their antidepressant effects (van Broekhoven \& Verkes, 2003), if a history of depressive illness contributes to long-term alterations in neuroactive steroid concentrations that persist beyond remission of the depressive episode, as the present results suggest, this may represent one mechanism contributing to the increased risk for recurrent depression in those with a history of depression (Lewinsohn, 1989). Future longitudinal studies assessing neuroactive steroid concentrations in remitted patients with histories of depression and subsequent depression episodes would be needed to address this possibility.

\section{Acknowledgments}

RO1-MH051246 (SSG); RO1-MH081837 (DRR and SSG); R37-AA10564 (ALM) and the North Carolina Biotechnology Center.

\section{References}

Ahrens T, Deuschle M, Krumm B, van der Pompe G, den Boer JA, Lederbogen F. Pituitary-adrenal and sympathetic nervous system responses to stress in women remitted from recurrent major depression. Psychosom Med. 2008; 70(4):461-467. [PubMed: 18378864] 
Andreen L, Bixo M, Nyberg S, Sundstrom-Poromaa I, Backstrom T. Progesterone effects during sequential hormone replacement therapy. Eur J Endocrinol. 2003; 148(5):571-577. [PubMed: 12720542]

Andreen L, Nyberg S, Turkmen S, van Wingen G, Fernandez G, Backstrom T. Sex steroid induced negative mood may be explained by the paradoxical effect mediated by GABAA modulators. Psychoneuroendocrinology. 2009; 34(8):1121-1132. [PubMed: 19272715]

Barbaccia ML, Roscetti G, Trabucchi M, Purdy RH, Mostallino MC, Concas A, et al. The effects of inhibitors of GABAergic transmission and stress on brain and plasma allopregnanolone concentrations. Br J Pharmacol. 1997; 120(8):1582-1588. [PubMed: 9113382]

Beauchamp MH, Ormerod BK, Jhamandas K, Boegman RJ, Beninger RJ. Neurosteroids and reward: allopregnanolone produces a conditioned place aversion in rats. Pharmacol Biochem Behav. 2000; 67(1):29-35. [PubMed: 11113481]

Beck AT, Ward CH, Mendelson M, Mock J, Erbaugh J. An inventory for measuring depression. Arch Gen Psychiatry. 1961; 4:561-571. [PubMed: 13688369]

Biggio, G.; Purdy, RH. International review of neurobiology. Vol. Vol. 46. New York: Academic Press; 2001. Neurosteroids and brain function.

Bjorn I, Bixo M, Nojd KS, Collberg P, Nyberg S, Sundstrom-Poromaa I, et al. The impact of different doses of medroxyprogesterone acetate on mood symptoms in sequential hormonal therapy. Gynecol Endocrinol. 2002; 16(1):1-8. [PubMed: 11915576]

Blanchard DC, Sakai RR, McEwen B, Weiss SM, Blanchard RJ. Subordination stress: behavioral, brain, and neuroendocrine correlates. Behav Brain Res. 1993; 58(1-2):113-121. [PubMed: 8136039]

De Geyter C, De Geyter M, Huber PR, Nieschlag E, Holzgreve W. Progesterone serum levels during the follicular phase of the menstrual cycle originate from the crosstalk between the ovaries and the adrenal cortex. Hum Reprod. 2002; 17(4):933-939. [PubMed: 11925385]

Deuschle M, Hamann B, Meichel C, Krumm B, Lederbogen F, Kniest A, et al. Antidepressive treatment with amitriptyline and paroxetine: effects on saliva cortisol concentrations. J Clin Psychopharmacol. 2003; 23(2):201-205. [PubMed: 12640223]

Dong E, Matsumoto K, Uzunova V, Sugaya I, Takahata H, Nomura H, et al. Brain 5alphadihydroprogesterone and allopregnanolone synthesis in a mouse model of protracted social isolation. Proc Natl Acad Sci U S A. 2001; 98(5):2849-2854. [PubMed: 11226329]

Eser D, Romeo E, Baghai TC, di Michele F, Schule C, Pasini A, et al. Neuroactive steroids as modulators of depression and anxiety. Neuroscience. 2006; 138(3):1041-1048. [PubMed: 16310959]

Freeman EW, Weinstock L, Rickels K, Sondheimer SJ, Coutifaris C. A placebo-controlled study of effects of oral progesterone on performance and mood. Br J Clin Pharmacol. 1992; 33(3):293-298. [PubMed: 1576050]

Frye CA, Duncan JE, Basham M, Erskine MS. Behavioral effects of 3 alpha-androstanediol. II: Hypothalamic and preoptic area actions via a GABAergic mechanism. Behav Brain Res. 1996; 79(1-2):119-130. [PubMed: 8883823]

Frye CA, Walf AA, Rhodes ME, Harney JP. Progesterone enhances motor, anxiolytic, analgesic, and antidepressive behavior of wild-type mice, but not those deficient in type 15 alpha-reductase. Brain Res. 2004; 1004(1-2):116-124. [PubMed: 15033426]

Gilbert Evans SE, Ross LE, Sellers EM, Purdy RH, Romach MK. 3alpha-reduced neuroactive steroids and their precursors during pregnancy and the postpartum period. Gynecol Endocrinol. 2005; 21(5):268-279. [PubMed: 16373246]

Gillespie CF, Nemeroff CB. Hypercortisolemia and depression. Psychosom Med. 2005; 67 Suppl 1:S26-S28. [PubMed: 15953796]

Girdler SS, Straneva PA, Light KC, Pedersen CA, Morrow AL. Allopregnanolone levels and reactivity to mental stress in premenstrual dysphoric disorder. Biol Psychiatry. 2001; 49(9):788-797. [PubMed: 11331087]

Guo AL, Petraglia F, Criscuolo M, Ficarra G, Nappi RE, Palumbo MA, et al. Evidence for a role of neurosteroids in modulation of diurnal changes and acute stress-induced corticosterone secretion in rats. Gynecol Endocrinol. 1995; 9(1):1-7. [PubMed: 7793294] 
Kavaliers M, Wiebe JP. Analgesic effects of the progesterone metabolite, 3 alpha-hydroxy- 5 alphapregnan-20-one, and possible modes of action in mice. Brain Res. 1987; 415(2):393-398. [PubMed: 3111642]

Kirschbaum C, Pirke KM, Hellhammer DH. The 'Trier Social Stress Test'--a tool for investigating psychobiological stress responses in a laboratory setting. Neuropsychobiology. 1993; 28(1-2):7681. [PubMed: 8255414]

Klatzkin RR, Morrow AL, Light KC, Pedersen CA, Girdler SS. Associations of histories of depression and PMDD diagnosis with allopregnanolone concentrations following the oral administration of micronized progesterone. Psychoneuroendocrinology. 2006a; 31(10):1208-1219. [PubMed: 17046166]

Klatzkin RR, Morrow AL, Light KC, Pedersen CA, Girdler SS. Histories of depression, allopregnanolone responses to stress, and premenstrual symptoms in women. Biol Psychol. 2006b; 71(1):2-11. [PubMed: 15951099]

Lewinsohn PM, Zeiss AM, Duncan EM. Probability of Relapse After Recovery From an Episode of Depression. Journal of Abnormal Psychology. 1989; 98(2):107-116. [PubMed: 2708651]

Majewska MD, Harrison NL, Schwartz RD, Barker JL, Paul SM. Steroid hormone metabolites are barbiturate-like modulators of the GABA receptor. Science. 1986; 232(4753):1004-1007. [PubMed: 2422758]

Maninger N, Capitanio JP, Mason WA, Ruys JD, Mendoza SP. Acute and chronic stress increase DHEAS concentrations in rhesus monkeys. Psychoneuroendocrinology.

McAuley JW, Kroboth FJ, Kroboth PD. Oral administration of micronized progesterone: a review and more experience. Pharmacotherapy. 1996; 16(3):453-457. [PubMed: 8726605]

McEwen BS. Protective and damaging effects of stress mediators. N Engl J Med. 1998; 338(3):171179. [PubMed: 9428819]

McKittrick CR, Blanchard DC, Blanchard RJ, McEwen BS, Sakai RR. Serotonin receptor binding in a colony model of chronic social stress. Biol Psychiatry. 1995; 37(6):383-393. [PubMed: 7772647]

Miczek KA, DeBold JF, van Erp AM, Tornatzky W. Alcohol, GABAA-benzodiazepine receptor complex, and aggression. Recent Dev Alcohol. 1997; 13:139-171. [PubMed: 9122494]

Miller GE, Chen E, Zhou ES. If it goes up, must it come down? Chronic stress and the hypothalamicpituitary-adrenocortical axis in humans. Psychol Bull. 2007; 133(1):25-45. [PubMed: 17201569]

Monroe SM, Harkness KL. Life Stress, the "Kindling" Hypothesis, and the Recurrence of Depression: Considerations From a Life Stress Perspective. Psychological Review. 2005; 112(2):417-445. [PubMed: 15783292]

Morrow AL. Recent developments in the significance and therapeutic relevance of neuroactive steroids--Introduction to the special issue. Pharmacol Ther. 2007; 116(1):1-6. [PubMed: 17531324]

Morrow AL, Devaud LL, Purdy RH, Paul SM. Neuroactive steroid modulators of the stress response. Ann N Y Acad Sci. 1995; 771:257-272. [PubMed: 8597405]

Naert G, Maurice T, Tapia-Arancibia L, Givalois L. Neuroactive steroids modulate HPA axis activity and cerebral brain-derived neurotrophic factor (BDNF) protein levels in adult male rats. Psychoneuroendocrinology. 2007; 32(8-10):1062-1078. [PubMed: 17928160]

Nappi RE, Petraglia F, Luisi S, Polatti F, Farina C, Genazzani AR. Serum allopregnanolone in women with postpartum "blues". Obstet Gynecol. 2001; 97(1):77-80. [PubMed: 11152912]

Patchev VK, Hassan AH, Holsboer DF, Almeida OF. The neurosteroid tetrahydroprogesterone attenuates the endocrine response to stress and exerts glucocorticoid-like effects on vasopressin gene transcription in the rat hypothalamus. Neuropsychopharmacology. 1996; 15(6):533-540. [PubMed: 8946427]

Paul SM, Purdy RH. Neuroactive steroids. Faseb J. 1992; 6(6):2311-2322. [PubMed: 1347506]

Porcu P, O'Buckley TK, Alward SE, Marx CE, Shampine LJ, Girdler SS, et al. Simultaneous quantification of GABAergic $3 \alpha, 5 \alpha / 3 \alpha, 5 \beta$ neuroactive steroids in human and rat serum. Steroids. 2009; 74:463-473. [PubMed: 19171160]

Prince MJ, Harwood RH, Blizard RA, Thomas A, Mann AH. Social support deficits, loneliness and life events as risk factors for depression in old age. The Gospel Oak Project VI. Psychol Med. 1997; 27(2):323-332. [PubMed: 9089825] 
Purdy RH, Morrow AL, Moore PH Jr, Paul SM. Stress-induced elevations of gamma-aminobutyric acid type A receptor-active steroids in the rat brain. Proc Natl Acad Sci U S A. 1991; 88(10): 4553-4557. [PubMed: 1852011]

Reddy DS. Physiological role of adrenal deoxycorticosterone-derived neuroactive steroids in stresssensitive conditions. Neuroscience. 2006; 138(3):911-920. [PubMed: 16325348]

Roberts RE, Kaplan GA, Shema SJ, Strawbridge WJ. Does growing old increase the risk for depression? Am J Psychiatry. 1997; 154(10):1384-1390. [PubMed: 9326820]

Romeo E, Strohle A, Spalletta G, di Michele F, Hermann B, Holsboer F, et al. Effects of antidepressant treatment on neuroactive steroids in major depression. Am J Psychiatry. 1998; 155(7):910-913. [PubMed: 9659856]

Sachar EJ, Hellman L, Fukushima DK, Gallagher TF. Cortisol production in depressive illness. A clinical and biochemical clarification. Arch Gen Psychiatry. 1970; 23(4):289-298. [PubMed: 4918519]

Serra M, Pisu MG, Littera M, Papi G, Sanna E, Tuveri F, et al. Social isolation-induced decreases in both the abundance of neuroactive steroids and $\mathrm{GABA}(\mathrm{A})$ receptor function in rat brain. $\mathrm{J}$ Neurochem. 2000; 75(2):732-740. [PubMed: 10899949]

Siever LJ, Davis KL. Overview: toward a dysregulation hypothesis of depression. Am J Psychiatry. 1985; 142(9):1017-1031. [PubMed: 2862799]

Simpson ER, Waterman MR. Regulation of the synthesis of steroidogenic enzymes in adrenal cortical cells by ACTH. Annu Rev Physiol. 1988; 50:427-440. [PubMed: 2837136]

Steiger A, von Bardeleben U, Herth T, Holsboer F. Sleep EEG and nocturnal secretion of cortisol and growth hormone in male patients with endogenous depression before treatment and after recovery. J Affect Disord. 1989; 16(2-3):189-195. [PubMed: 2522118]

Strohle A, Pasini A, Romeo E, Hermann B, Spalletta G, di Michele F, et al. Fluoxetine decreases concentrations of 3 alpha, 5 alpha-tetrahydrodeoxycorticosterone (THDOC) in major depression. $\mathbf{J}$ Psychiatr Res. 2000; 34(3):183-186. [PubMed: 10867112]

Strohle A, Romeo E, Hermann B, Pasini A, Spalletta G, di Michele F, et al. Concentrations of 3 alphareduced neuroactive steroids and their precursors in plasma of patients with major depression and after clinical recovery. Biol Psychiatry. 1999; 45(3):274-277. [PubMed: 10023501]

Strous RD, Maayan R, Weizman A. The relevance of neurosteroids to clinical psychiatry: from the laboratory to the bedside. Eur Neuropsychopharmacol. 2006; 16(3):155-169. [PubMed: 16257183]

Uzunova V, Sampson L, Uzunov DP. Relevance of endogenous 3alpha-reduced neurosteroids to depression and antidepressant action. Psychopharmacology (Berl). 2006; 186(3):351-361. [PubMed: 16249906]

Uzunova V, Sheline Y, Davis JM, Rasmusson A, Uzunov DP, Costa E, et al. Increase in the cerebrospinal fluid content of neurosteroids in patients with unipolar major depression who are receiving fluoxetine or fluvoxamine. Proc Natl Acad Sci U S A. 1998; 95(6):3239-3244. [PubMed: 9501247]

Vallee M, Rivera JD, Koob GF, Purdy RH, Fitzgerald RL. Quantification of neurosteroids in rat plasma and brain following swim stress and allopregnanolone administration using negative chemical ionization gas chromatography/mass spectrometry. Anal Biochem. 2000; 287(1):153166. [PubMed: 11078595]

van Broekhoven F, Verkes RJ. Neurosteroids in depression: a review. Psychopharmacology (Berl). 2003; 165(2):97-110. [PubMed: 12420152]

Zeger SL, Liang KY, Albert PS. Models for longitudinal data: a generalized estimating equation approach. Biometrics. 1988; 44(4):1049-1060. [PubMed: 3233245] 


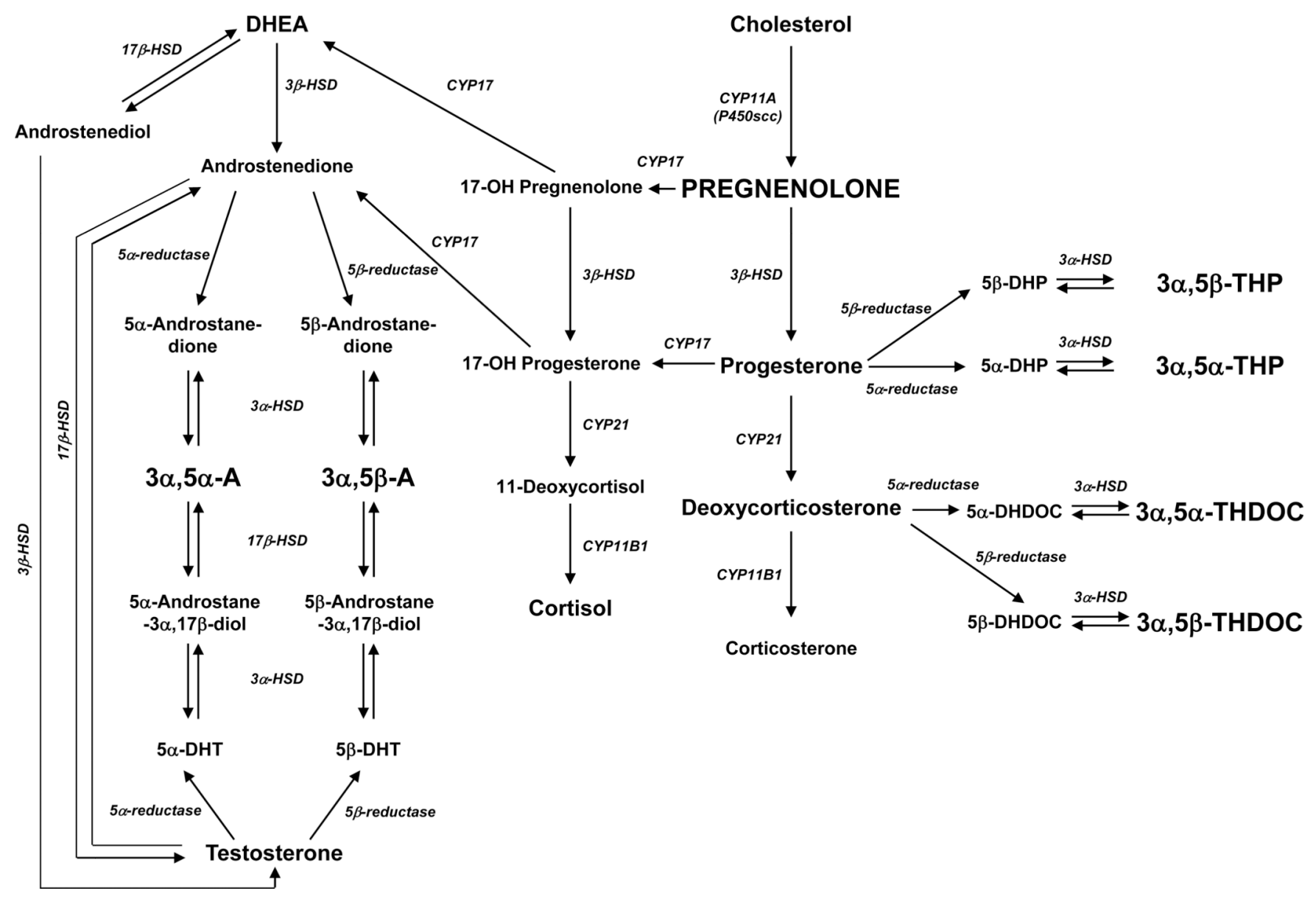

Figure 1.

Biosynthetic pathway for the GABAergic neuroactive steroids measured herein (indicated in

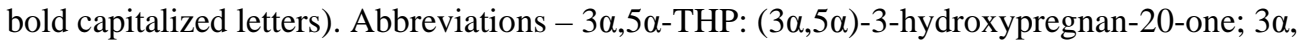

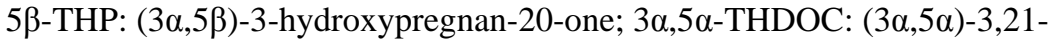

dihydroxypregnan-20-one; $3 \alpha, 5 \beta$-THDOC: $(3 \alpha, 5 \beta)$-3,21-dihydroxypregnan-20-one; $3 \alpha, 5 \alpha$ A: $(3 \alpha, 5 \alpha)$-3-hydroxyandrostan-17-one; $3 \alpha, 5 \beta-A$ : $(3 \alpha, 5 \beta)$-3-hydroxyandrostan-17-one; $5 \alpha$ DHP: $5 \alpha$-dihydroprogesterone; $5 \beta$-DHP: $5 \beta$-dihydroprogesterone; $5 \alpha$-DHDOC: $5 \alpha$ dihydrodeoxycorticosterone; $5 \beta$-DHDOC: $5 \beta$-dihydrodeoxycorticosterone; $5 \alpha$-DHT: $5 \alpha$ dihydrotestosterone; $5 \beta$-DHT: $5 \beta$-dihydrotestosterone; DHEA: dehydroepiandrosterone; HSD: hydroxysteroid dehydrogenase. 

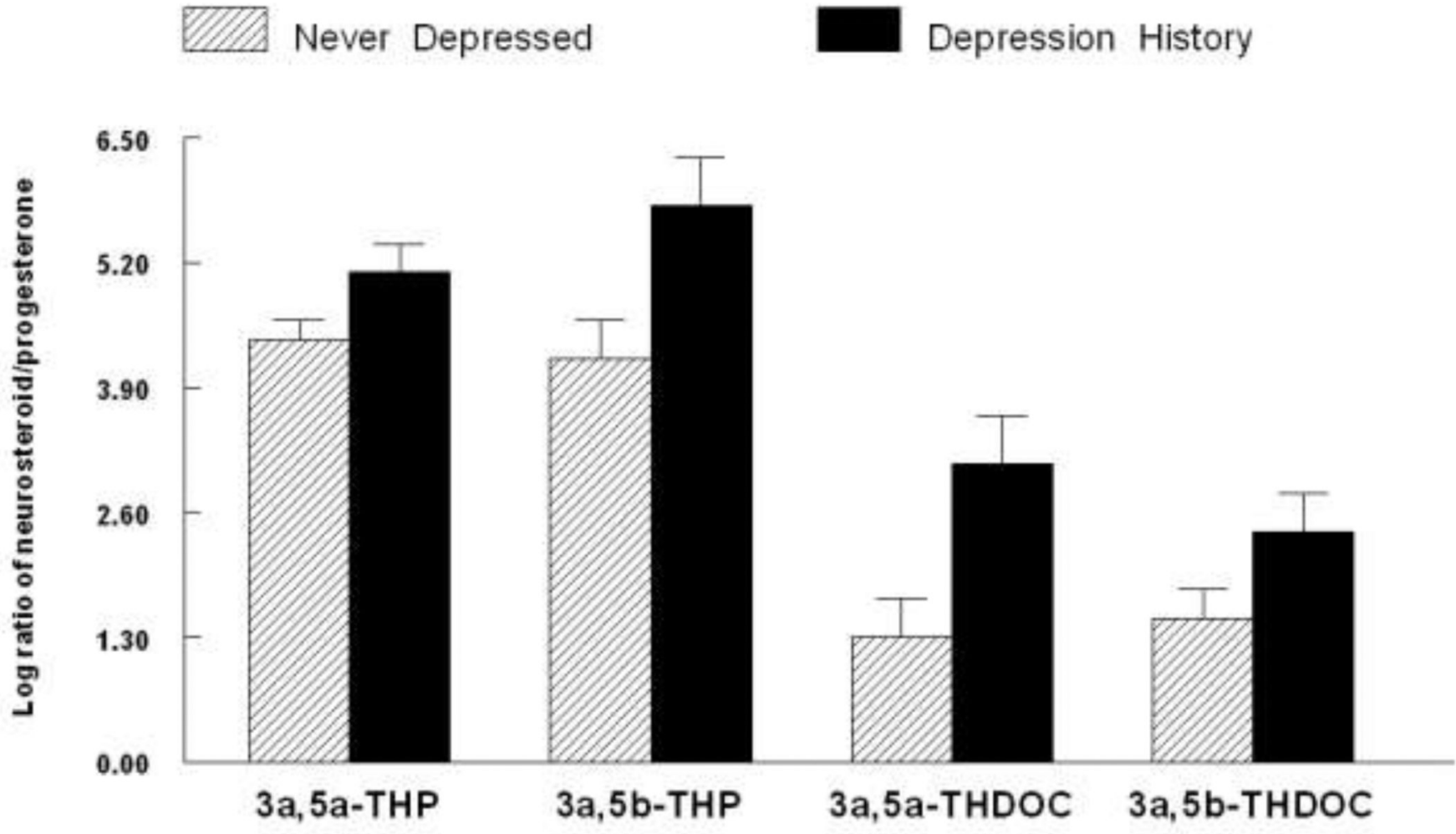

Figure 2.

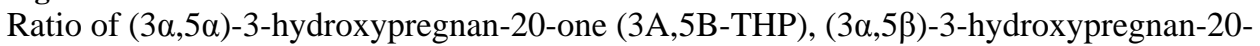
one (3A,5B-THP), $(3 \alpha, 5 \alpha)-3,21$-dihydroxypregnan-20-one (3A,5B-THDOC), and ( $3 \alpha$, $5 \beta)-3,21$-dihydroxypregnan-20-one (3A,5B-THDOC) to progesterone in women as a function of depression histories. 
Table 1

Mean (+SD) baseline and demographic factors as a function of prior depression

\begin{tabular}{|c|c|c|}
\hline & $\begin{array}{c}\text { Never Depressed } \\
(\mathbf{n}=\mathbf{1 7})\end{array}$ & $\begin{array}{c}\text { History of Depression } \\
(\mathbf{n}=\mathbf{1 1})\end{array}$ \\
\hline Age & $33.6(6.9)$ & $37(5.6)$ \\
\hline BMI & $25.3(5.0)$ & $24.5(3.6)$ \\
\hline No. Minorities (\%) & $3(17.6 \%)$ & $2(18.2 \%)$ \\
\hline No. PTSD (\%) & $0(0 \%)$ & $0(0 \%)$ \\
\hline No. Eating Disorders (\%) & $0(0 \%)$ & $1(9.1 \%)$ \\
\hline No. Substance Abuse (\%) & $1(6 \%)$ & $4(36.4 \%)$ \\
\hline No. Prior Anxiety (\%) & $0(0 \%)$ & $3(27.2 \%)$ \\
\hline Beck Depression Inventory Score & $2.8(3.2)$ & $6.2(4.4)$ \\
\hline Months Since Last Depressive Episode & & $60.4(10.9)--$ \\
\hline No. Depressive Episodes: & & $5(45 \%)$ \\
Single (\%) & & $-5(55 \%)$ \\
Multiple (\%) & & \\
\hline \multicolumn{2}{|c|}{} & \\
\hline
\end{tabular}

$\mathrm{BMI}=$ Body Mass Index,

A Prior DEP > no prior DEP, $p<.05$ 


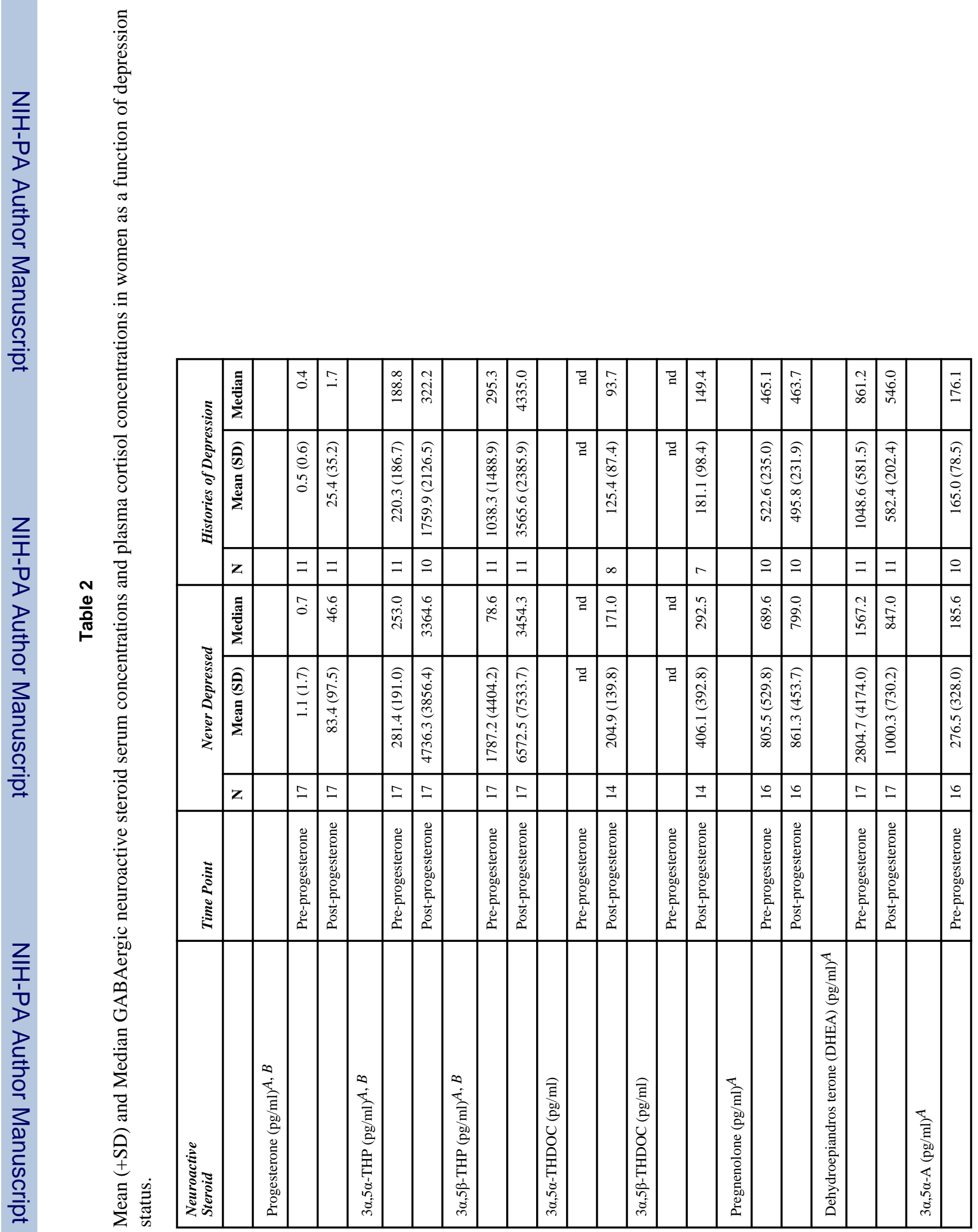




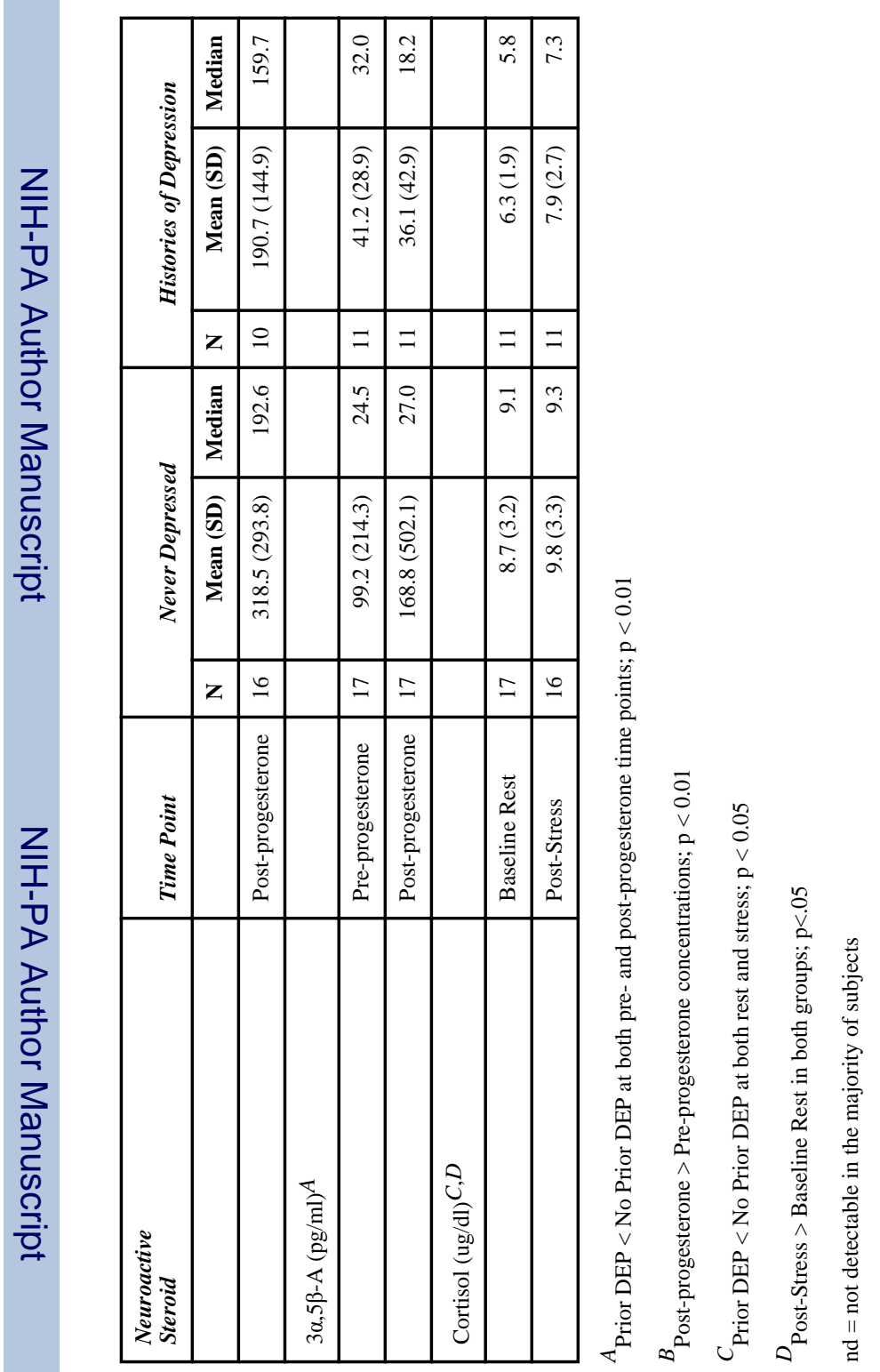

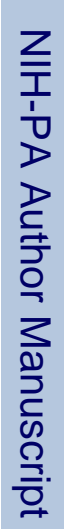

